\title{
Article \\ Behavior of Generated Gas during Femtosecond Laser Lens Irradiation in Porcine Cadaver Eyes
}

\author{
Yoichiro Masuda ${ }^{1, *(\mathbb{D})}$, Kotaro Oki $^{2}$, Akira Watanabe ${ }^{1}$, Makiko Ohkido ${ }^{3}$, Hisaharu Iwaki ${ }^{4}$, Takuya Shiba ${ }^{5}$ \\ and Tadashi Nakano ${ }^{1}$ \\ 1 Department of Ophthalmology, School of Medicine, The Jikei University, Tokyo 105-8461, Japan; \\ akirawa@jikei.ac.jp (A.W.); tnakano@jikei.ac.jp (T.N.) \\ 2 Oki Eye Surgery Center, Tokyo 171-0014, Japan; kotaro-o@pc4.so-net.ne.jp \\ 3 Department of Molecular Biology, School of Medicine, The Jikei University, Tokyo 105-8461, Japan; \\ mohkido@jikei.ac.jp \\ 4 Iwaki Eye Clinic, Tokyo 131-0032, Japan; iwakieyeclinic@nifty.com \\ 5 Roppongi Shiba Eye Clinic, Tokyo 106-0032, Japan; shiba@rb3.so-net.ne.jp \\ * Correspondence: ymasuda@jikei.ac.jp; Tel.: +81-3-3433-1111
}

check for

updates

Citation: Masuda, Y.; Oki, K.;

Watanabe, A.; Ohkido, M.; Iwaki, H.;

Shiba, T.; Nakano, T. Behavior of

Generated Gas during Femtosecond

Laser Lens Irradiation in Porcine

Cadaver Eyes. Appl. Sci. 2021, 11

8247. https://doi.org/10.3390/

app11178247

Academic Editors: Zhenxu Bai,

Qiang Wu and Quan Sheng

Received: 30 July 2021

Accepted: 2 September 2021

Published: 6 September 2021

Publisher's Note: MDPI stays neutral with regard to jurisdictional claims in published maps and institutional affiliations.

Copyright: (c) 2021 by the authors. Licensee MDPI, Basel, Switzerland. This article is an open access article distributed under the terms and conditions of the Creative Commons Attribution (CC BY) license (https:// creativecommons.org/licenses/by/ $4.0 /)$.

\begin{abstract}
Background: We investigated the behavior of gas inside a lens and its influence on the lens capsule, which may cause complications by lens irradiation with a femtosecond laser cataract surgery device. (2) Methods: The crystalline lenses of 6-month-old porcine cadaver eyes were observed during laser irradiation. An intraocular endoscope in the vitreous cavity was used to measure the posterior capsule position. Optical coherence tomography measurements of the anterior chamber depth before and after the laser irradiation, as well as measurements of the equatorial perimeter of the extracted lens, were compared with those of the controls. (3) Results: Femtosecond laser-generated gas in the porcine lens was dependent on laser irradiation energy. Increases in the amount of laser irradiation energy caused the generated gas to coalesce, move backwards beyond the laser irradiation site, and expand the lens capsule and posterior capsule. (4) Conclusions: The present results suggest that laser irradiation-induced gas moves in the direction of the posterior capsule beyond the lens irradiation site and expands the lens capsule, which may be involved in the development of capsular block syndrome.
\end{abstract}

Keywords: femtosecond laser-assisted cataract surgery; FLACS; capsular block syndrome; laser-induced gas; posterior capsule rupture

\section{Introduction}

Femtosecond laser-assisted cataract surgery (FLACS) irradiates an extremely short femtosecond pulse laser, thereby performing minimally invasive and accurate cataract surgery without causing shock or heat [1]. By irradiating the laser as if to create perforations, we allow adjacent perforations to connect to each other. We repeat this process, and perform anterior capsulotomy and lens fragmentation. Depending on the distance between two adjacent perforations, and the setting of each laser energy amount, the eye can be irradiated with a variety of designs. Depending on the design, the amount of total laser irradiation energy is determined. An important difference from conventional manual cataract surgery (CCS) is that gas generation occurs within the lens during the laser irradiation, which is laser irradiation-energy-dependent $[2,3]$. Thus, there is a higher risk for laser-induced gas to increase intracapsular pressure and cause intraoperative capsular block syndrome (CBS), leading to posterior capsule rupture during FLACS as compared to CCS $[1,3,4]$. Posterior capsule rupture during cataract surgery is a complication that must be avoided because it prevents the intraocular lens from being inserted into the capsule. However, the behavior of the generated gas inside the lens during femtosecond laser irradiation and the effect on the lens capsule are yet to be well understood. The risk of capsule rupture 
due to the CBS could be related to the properties of laser-induced gas distribution and the lens capsule dilation caused by gas pressure. Therefore, the aim of this study was to investigate the behavior of the femtosecond laser-induced gas inside the lens and then determine its influence on the lens capsule. In order to achieve this objective, we observed porcine crystalline lenses during laser irradiation and measured the posterior capsule position (PCP) by using an intraocular endoscope in the vitreous cavity. Furthermore, optical coherence tomography (OCT) was used to measure the anterior chamber depth (ACD) before and after laser irradiation, and after measuring the equatorial perimeter of the extracted lenses; values were then compared with untreated porcine lenses as controls. The present results suggested that there was inflation of the laser irradiation-induced gas in the direction of the posterior capsule beyond the lens irradiation site along with expansion of the lens capsule, which could be involved in the development of CBS.

\section{Materials and Methods}

The ethics committee of Jikei University ruled that approval was not required for the study.

Porcine eyes: This study used 6-month-old porcine cadaver eyes $(\mathrm{N}=26)$ that had been obtained from a local abattoir and stored at $4{ }^{\circ} \mathrm{C}$. All eyes were used within $12 \mathrm{~h}$ of enucleation. Out of the 26 total eyes, 16 were laser-irradiated, while 10 were used as controls.

Femtosecond laser lens irradiation procedure: We used femtosecond laser cataract surgical system CATALYS (Johnson \& Johnson Surgical Vision Inc., Santa Ana, CA, USA), which includes the use of laser irradiation for lens fragmentation and quadrants. After connecting the liquid optic interface (and endoscope) to the porcine eye (Figure 1), we manually customized the anatomic dimensions (e.g., anterior and posterior corneal curvature, anterior and posterior capsule). Since the thickness of the porcine lens made it impossible to detect the posterior capsule when using the installed OCT, the tentative PCP was manually set so that lens thickness was $6.5 \mathrm{~mm}$. Depending on the distance between two adjacent perforations and the setting of each laser energy amount, the laser could be irradiated with a variety of designs. Depending on the design, the CATALYS system determines the total amount of laser energy to be delivered. In order to evaluate the influence of the emerging gas bubbles, we varied the lens fragmentation setting (energy of lens fragmentation) for each set of experiments (Table 1).

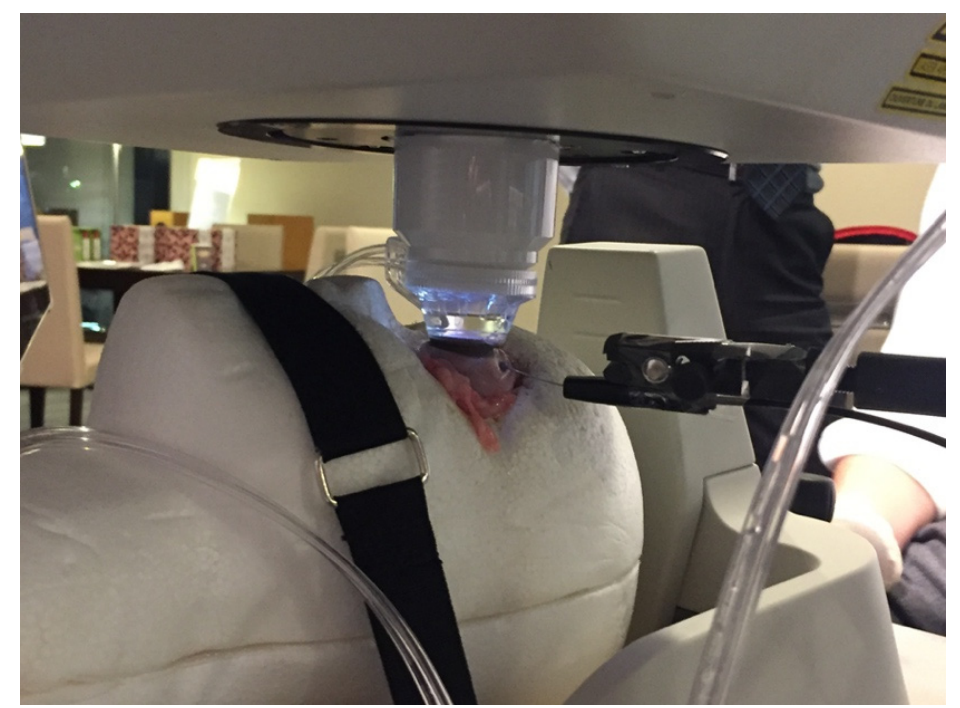

Figure 1. Eyeballs were placed on custom-made stands in order to achieve an appropriate height and position for femtosecond laser system CATALYS for the purpose of connecting the liquid optic interface and endoscope to the porcine eye. 
Table 1. Lens fragmentation setting using femtosecond laser surgical system CATALYS.

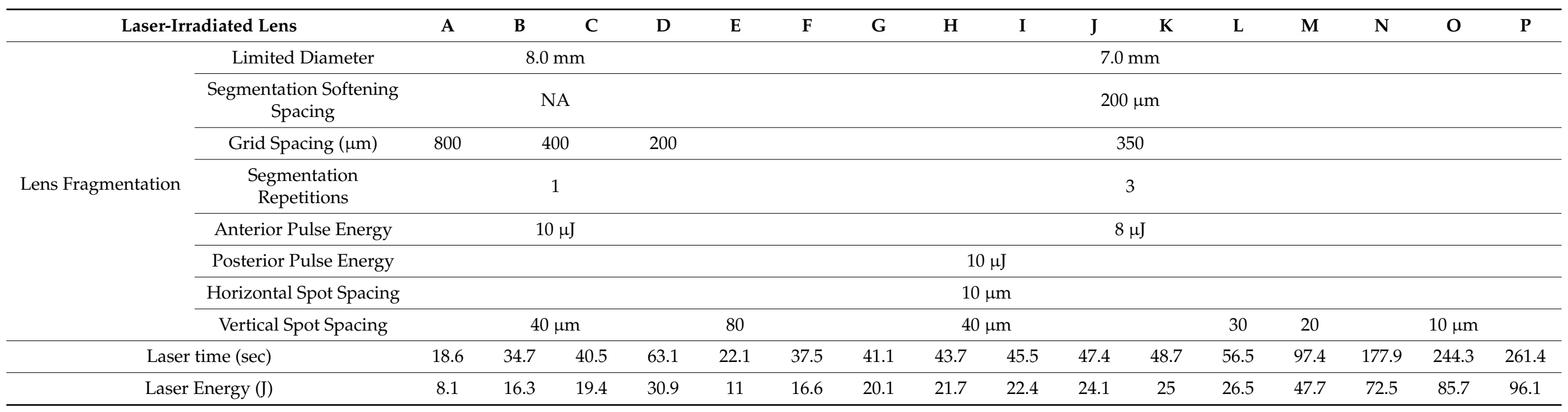


Anterior chamber depth measurement: The installed OCT was used to measure the ACD pre- and post-laser irradiation in 5 eyes (lenses E, G, H, and I in Table 1). The OCT measurement after laser irradiation took less than $1 \mathrm{~min}$. ACD correlations between preand post-laser irradiation were statistically analyzed by a Wilcoxon signed-rank test using IBM-SPSS software (SPSS, Inc., Chicago, IL, USA). The ACD change ratio was calculated by dividing the ACD after laser irradiation by the ACD before laser irradiation. The correlation between ACD change ratio and laser total energy was statistically analyzed by a simple regression analysis using IBM-SPSS software.

Endoscopic imaging and lens posterior capsule position measurement: Before customizing for anatomic dimensions, a 23-gauge trocar cannula (AU-1272EA2-06 DORC, Netherlands) was inserted into 6 eyes (F, G, H, I, K, P in Table 1) at a site that was located $6 \mathrm{~mm}$ from the corneal limbus. A 23-gauge intraocular endoscope (AS-611: Fiber Tech, Tokyo, Japan) that was connected to a 3 LED light-source device (FL-301: Fiber Tech, Tokyo, Japan) and 3 CMOS HD camera (FC-304: Fiber Tech, Tokyo, Japan) was inserted via the trocar, and then fixed to make it possible to image the back of the lens including the posterior capsule (Figure 1). Imaging of the posterior site of the lens was performed using this endoscopic device during the femtosecond laser irradiation of the lens.

After obtaining the endoscopic image, a graph of the ratio of the position of the posterior capsule per each $5 \mathrm{~s}$ of laser irradiation was prepared. Calculations were performed on the basis of the assumption that 1 was the value obtained by subtracting the PCP before laser irradiation from the postmaximal inflated capsule position. These values were defined as the PCP ratio. Since the laser irradiation time in the five lenses that did not rupture due to the laser irradiation differed among lenses, the graph is shown as the normalized time graph with a total irradiation time of 1 . After determining the regression line for the prepared graph, the coefficient of determination was calculated.

Microscopic imaging and lens-equatorial-perimeter measurement: After fragmentation and quadrants of the lens by femtosecond laser irradiation, the lens was extracted and imaged using a microscope (SMZ 18: Nikon, Tokyo, Japan). After excluding eyes destroyed by irradiation or during lens extraction, the equatorial perimeter of the crystalline lens in 10 eyes (lens A, C, D, E, G, H, I, J, K, L in Table 1) was measured using Image Processing and Analysis in JAVA program (Image J, National Institute of Health, Washington, DC, USA) and then compared with the control lenses from 10 eyes. The equivalence of the equatorial perimeter between control and laser-irradiated lenses was statistically analyzed by a Mann-Whitney U test using IBM-SPSS software. Correlation between the equatorial perimeter of the laser-irradiated lens and total laser energy was statistically analyzed by simple regression analysis using IBM-SPSS software.

\section{Results}

\subsection{Anterior Chamber Depth Measurement}

OCT determined that the average and standard deviation (SD) for the ACD before laser irradiation in five eyes was $2.66 \pm 0.11 \mathrm{~mm}$ (range, $2.5-2.8 \mathrm{~mm}$ ), while the ACD after laser irradiation was $2.4 \pm 0.16 \mathrm{~mm}$ (range, 2.2-2.6 mm). There was a significant difference observed between these two groups $(p=0.042<0.05)$ (Figure 2a). However, in the scope of this study, there was no correlation between laser irradiation energy dose and ACD change ratio $(p=0.054>0.05)$ (Figure $2 b)$. These results indicate that, although the laser irradiation caused the ACD to become significantly shallower, there was no correlation with the amount of the laser irradiation energy in the scope of this study. 

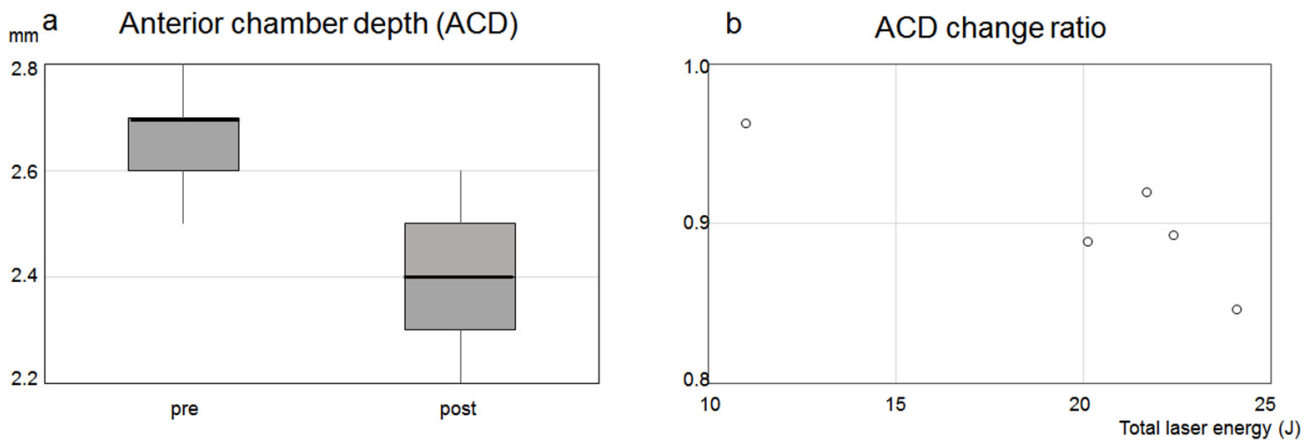

Figure 2. Although laser irradiation caused anterior chamber depth to become significantly shallower, there was no correlation with the amount of laser irradiation energy. (a) Box-plot diagram showing mean values of anterior chamber depth in the eye before and after laser irradiation. Boxes, interquartile range (difference between upper 75\% and lower $25 \%$ quartiles); thick black lines, median; whiskers, highest and lowest values that were not outliers or extreme values. A significant difference was observed in ACD before and after laser irradiation $(p=0.042<0.05)$ ). (b) There was no correlation between laser irradiation energy dose and ACD change ratio $(p=0.054>0.05)$.

\subsection{Endoscopic Imaging}

When using the endoscope to observe behind the crystalline lens during laser irradiation (Figure 1), images showed that the gas generated by the laser irradiation coalesced and expanded rearward beyond the irradiation site, leading to the expansion of the posterior capsule (Figures $3 a-d$ and $4 a, b$ ). The greater the amount of laser irradiation energy, the greater the amount of gas that was produced.
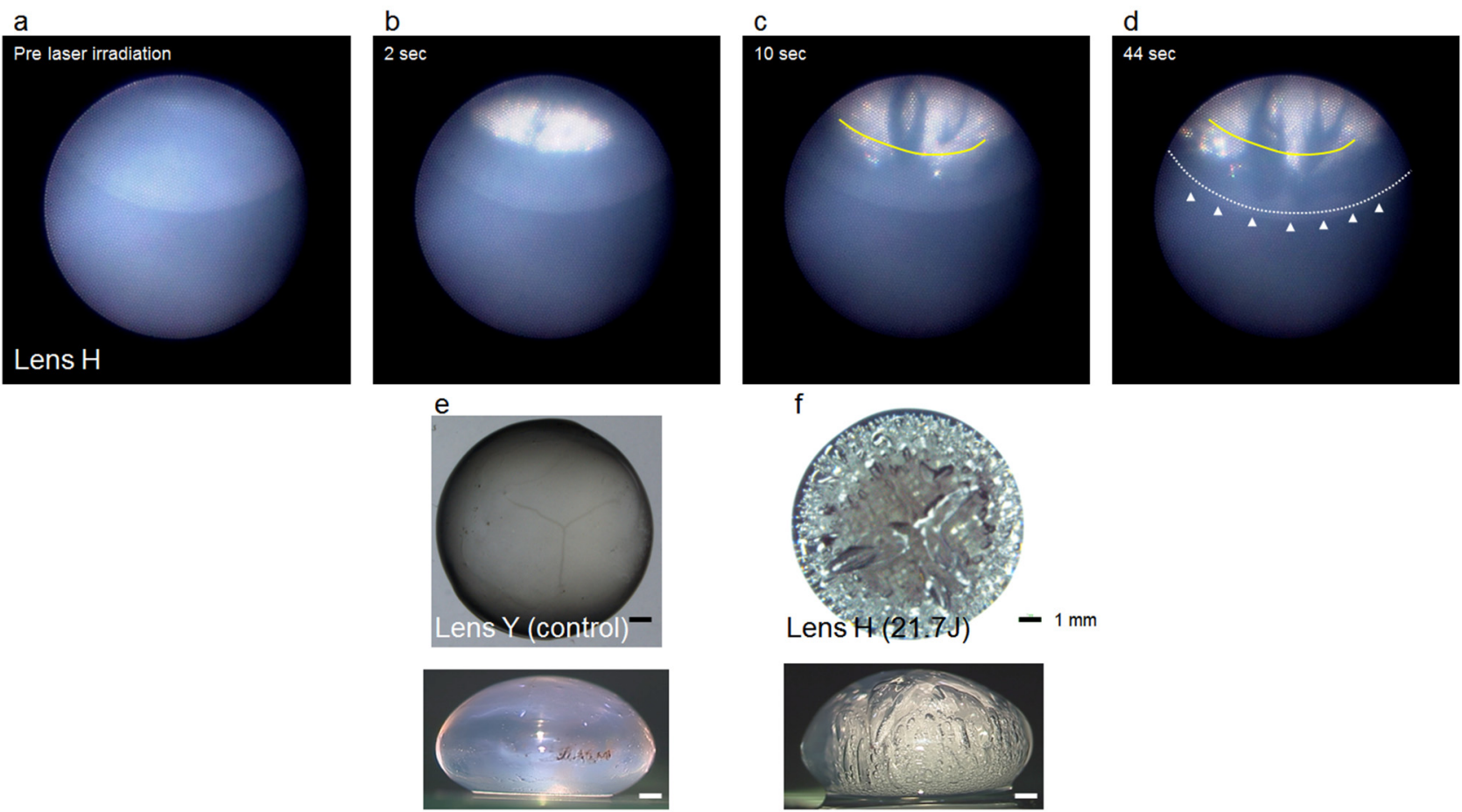

Figure 3. Generated gas by laser irradiation coalesced and moved rearward and beyond the irradiation site, thereby causing the posterior capsule to expand. Excised lens that was irradiated by the laser was expanded as compared to the control lens. (a-d) Endoscopic image after laser irradiation in lens H. (a) Before laser irradiation. (b) At $2 \mathrm{~s}$ after laser irradiation. (c) After $10 \mathrm{~s}$ of laser irradiation. (d) At $44 \mathrm{~s}$ after laser irradiation. Yellow dotted line indicates the starting position of laser irradiation; white dotted line indicates the posterior capsule position before laser irradiation; white arrowheads indicate posterior capsule position after expansion. (e,f) Microscopic image of extracted lens. (e) Control lens Y. (f) Lens H after laser irradiation. Scale bar is $1 \mathrm{~mm}$. Laser irradiation energy amount listed in parentheses. 


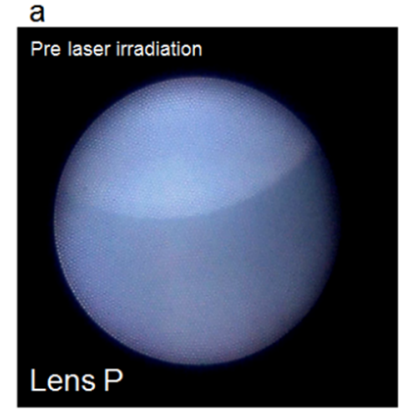

d

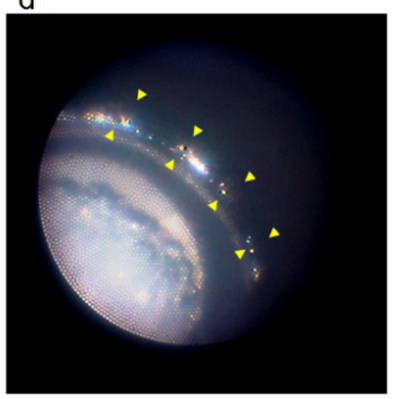

b

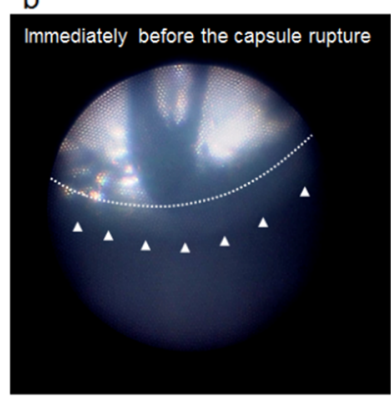

e

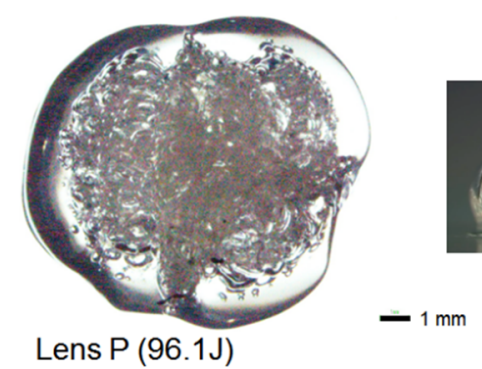

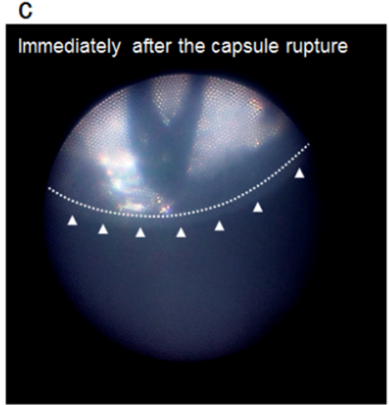

f

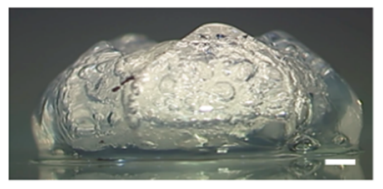

$\mathrm{mm}$

Figure 4. The greater the laser irradiation energy was, the more gas was produced. If pressure exceeded maximal pressure levels of the capsule, it ruptured. (a-c) Endoscopic images of lens $\mathrm{P}$ during laser irradiation procedure. (a) Before laser irradiation. (b) Immediately before rupture by CBS during laser irradiation. (c) Immediately after rupture by CBS during laser irradiation. (d) Endoscopic images of lens $\mathrm{P}$ from the posterior pole of the eye after laser irradiation. White dotted line indicates capsule position before laser irradiation; white arrowheads indicate posterior position after expansion; yellow arrowheads indicate gas distribution after bursting into the vitreous cavity behind the ciliary body. (e,f) Microscopic image of extracted lens P. Error bar is $1 \mathrm{~mm}$. Laser irradiation energy amount listed in parentheses.

When the pressure exceeded the maximal pressure level of the capsule, the capsule fractured (Figure 4c-f, Video S1). The excised lens, which was irradiated with the laser, was expanded as compared to the control lens (Figure 3e,f). The crystalline lens achieved buoyancy due to the generated gas; if the buoyancy overcame the weight of the lens, the lens was able to float in the saline (Figure 5).

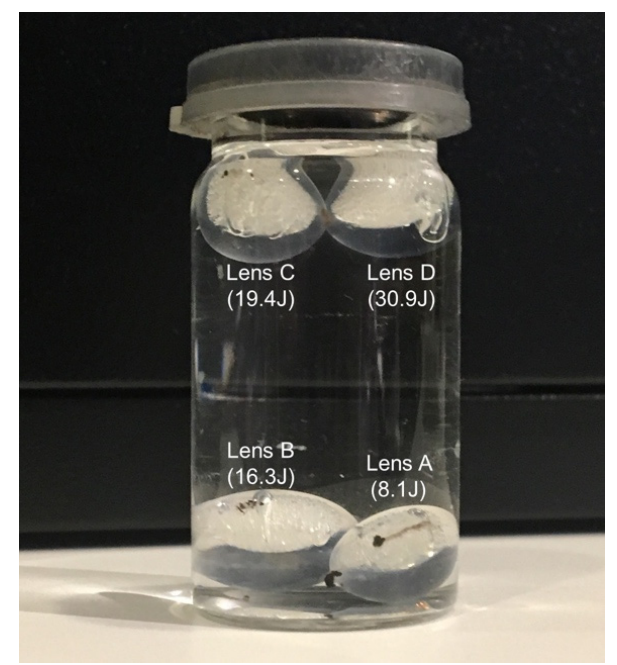

Figure 5. Generated gas caused the crystalline lens to become buoyant and overcome the weight of the lens, thereby allowing for it to float in the saline. Laser irradiation energy amount listed in parentheses. 


\subsection{Lens Posterior Capsule Position Measurement}

Figure 6a shows the PCP ratio obtained from the endoscopic imaging of five eyes (total laser energy amount of 16.6-25 J, laser irradiation time of 37.5-48.7 s). The coefficient of determination (R2) for each regression line was $0.938-0.981$. The PCP ratio within this irradiation time correlated well with laser irradiation time and laser energy amount (as the laser irradiation energy amounts were dependent on irradiation time). In lens $\mathrm{P}$, which was irradiated for $261 \mathrm{~s}$, the PCP ratio linearly increased up to about $70 \mathrm{~s}$ ( $22=0.981$ for the regression line up to $70 \mathrm{~s}$ ). Subsequently, the slope decreased, with bursts at $240 \mathrm{~s}$, followed by an abrupt rise in the PCP (Figures $6 \mathrm{~b}$ and $4 \mathrm{c}$ ). Analysis of the images showed that the posterior capsule tended to expand depending on laser irradiation time and energy amount.

a Posterior capsule position ratio

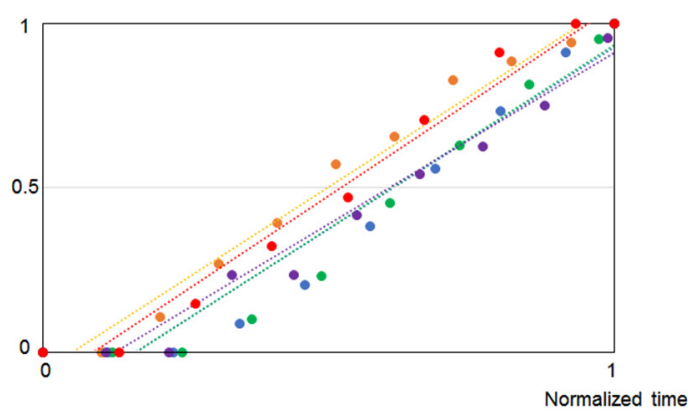

b PCP ratio

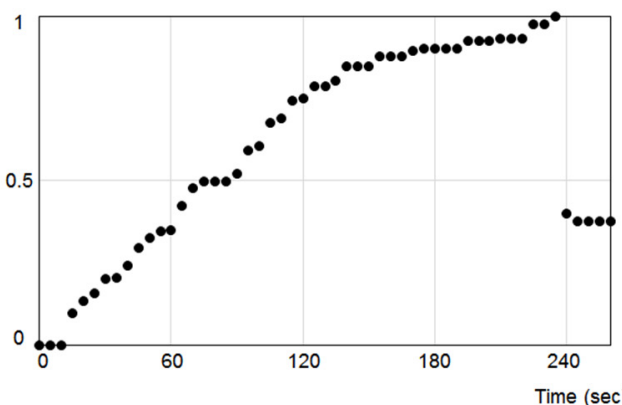

Figure 6. The PCP ratio correlated well with the laser irradiation time and laser energy amount. (a): The PCP ratio of 5 eyes (total laser energy amount 16.6-25 J, laser irradiation time 37.5-48.7 s). The coefficient of determination (R2) for each regression line was $0.938-0.981$. The PCP ratio within this irradiation time correlated well with the laser irradiation time and laser energy amount. (b): The PCP ratio in lens P, which was irradiated for $261 \mathrm{~s}$, linearly increased up to about $70 \mathrm{~s}$ $(\mathrm{R} 2=0.981)$, with the slope then decreasing, followed by a burst at $240 \mathrm{~s}$.

\subsection{Equatorial Perimeter Measurement}

For the 10 eyes used as controls, the average and SD for the equatorial perimeter of these lenses was $33.5 \pm 0.75 \mathrm{~mm}$ (range 32.5 to $34.8 \mathrm{~mm}$ ), while the mean and SD for the equatorial perimeter of the laser-irradiated lenses was $35.3 \pm 0.71 \mathrm{~mm}$ (range 34.3 to $36.7 \mathrm{~mm})$. There was a significant difference between these two groups $(p=0.001<0.05)$ (Figure 7a). However, there was no correlation found between the amount of laser irradiation energy and the equatorial perimeter for the laser-irradiated lenses $(p=0.899>0.05)$ (Figure $7 \mathrm{~b}$ ). These results indicate that while laser irradiation caused the average equatorial perimeter to be significantly longer, there was no correlation with the amount of the laser irradiation energy.

In summary, the femtosecond laser-generated gas in the porcine lens was dependent on the amount of laser irradiation energy. The generated gas coalesced and swelled backwards beyond the laser irradiation site, thereby expanding the lens capsule. The posterior capsule expansion was dependent upon the amount of laser irradiation energy. However, the ACD and equatorial perimeter were not dependent on the amount of laser energy. This suggests that the increased internal pressure due to the generated gas tended to significantly dilate the posterior capsule. 

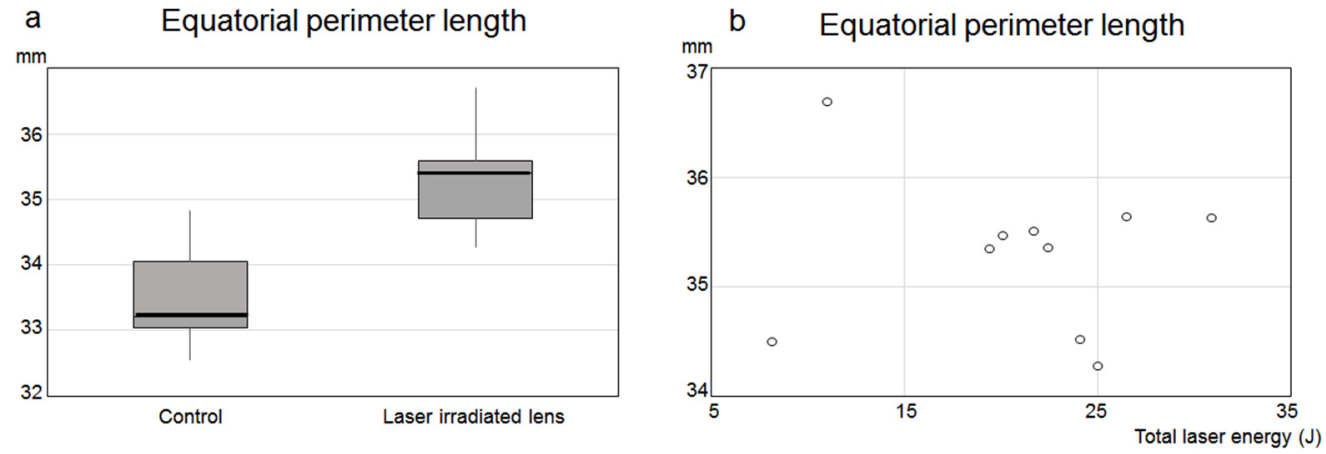

Figure 7. Although the laser irradiation caused significantly longer average equatorial perimeter, there was no correlation with the amount of laser irradiation energy. (a) Box-plot diagram showing mean values of equatorial perimeter length in control lens and laser-irradiated lens. Boxes, interquartile range (difference between upper 75\% and lower 25\% quartiles); thick black lines, median; whiskers, highest and lowest values that were not outliers or extreme values. There was significant difference between these two groups $(p=0.001<0.05)$. (b) No correlation was found between amount of laser irradiation energy and equatorial perimeter of laser-irradiated lens $(p=0.899>0.05)$.

\section{Discussion}

The present results suggest that femtosecond laser lens irradiation generates gas that expands towards the posterior capsule in conjunction with the amount of laser energy, which subsequently leads to the expansion of the posterior capsule. Although the sample size was small, the reason for not finding correlation between ACD change ratio and the amount of laser irradiation energy may be related to the fact that gas distribution is predominantly on the posterior capsule side, and the difference in distensibility between anterior and posterior capsules. During laser irradiation, generated gas causes an increase in the IOP by raising the lens capsule's internal pressure, with the increase in the IOP dependent on the amount of the laser energy [5]. The increases in the lens capsule's internal pressure caused by the generated gas can lead to intraoperative CBS. Although the laser irradiation settings used in this experiment were within the range that can be set in usual surgery, as the amount of laser energy increased, this led to an excessive amount of generated gas and potential ruptures in the capsule due to the occurrence of CBS during laser irradiation. Therefore, laser irradiation needs to be set at a level that avoids the generation of excessive gas. In addition, careful attention needs to be paid to intraoperative CBS avoidance to prevent pressure-handling issues that can occur in procedures such as hydrodissection, in which there could be an increase in inner lens capsule pressure after laser irradiation $[3,6,7]$.

This study focused on the behavior of gas found inside the lens capsule, which is the visible part of the generated gas, along with examining its influence on the lens capsule. However, $\mathrm{pH}$ acidification occurs in the aqueous humor due to the invisible part of the gas (carbon dioxide) [8]. This finding suggests that FLACS may influence ocular tissue due to currently unknown chemical changes and generated substances [9]. Since these changes are not reported for conventional cataract surgeries, additional investigations into these issues need to be undertaken.

There are several limitations in this study. First, it is possible that the size of the crystalline lens of the porcine eye may not be the same for individuals, and between the left and right eyes. However, it is difficult to accurately measure changes in lens diameter before and after laser irradiation, as laser crystalline lens irradiation by a clinical FLACS device cannot be performed after extracting a crystalline lens and accurately measuring the lens diameter. Second, in order to observe the extracted lens in this experiment, lens fragmentation was performed without performing anterior capsulotomy. If anterior capsulotomy had been performed, the behavior of the gas and the amount of energy that causes the capsule to burst might have been different from that observed in the actual experiment. However, since a large amount of the gas was distributed towards the 
back during actual clinical FLACS in combination with anterior capsulotomy and lens fragmentation, the phenomenon in this experiment was not wrong as a whole.

In summary, femtosecond laser porcine lens irradiation generated gas in the lens, with the extent of this increase dependent on the amount of laser irradiation energy. The generated gas moved beyond the irradiation site and expanded the posterior lens capsule. If the capsular pressure exceeded the maximal pressure levels, this could cause a capsule rupture. Thus, since there was an increase in capsular pressure due to the gas generated after femtosecond laser irradiation, careful attention needs to be paid to the laser irradiation setting and procedures such as hydrodissection in order to prevent further intracapsular pressurization and the possibility of capsule rupture.

Supplementary Materials: The following are available online at https:/ / www.mdpi.com/article/ 10.3390/app11178247/s1. Video S1: Endoscopic video (center) and CATALYS screen movie (inset top right) for the laser irradiation of lens P. After laser irradiation, the generated gas coalesced and expanded beyond the laser irradiation site to the rear. Gas production was dependent on laser irradiation time and energy amount. The lens capsule ruptured at $4 \mathrm{~min}$ after laser irradiation, with the posterior capsule sharply rising (laser-induced CBS). Since gas movement into the anterior chamber was not observed after the rupture, it was inferred that the rupture occurred in the posterior capsule.

Author Contributions: Conceptualization, Y.M. and K.O.; methodology, Y.M., K.O. and A.W.; validation, Y.M., K.O., and A.W.; formal analysis, Y.M.; investigation, Y.M., K.O., A.W. and M.O.; resources, H.I. and T.N.; data curation, Y.M.; writing—original draft preparation, Y.M.; writing-review and editing, Y.M., K.O., A.W., M.O., H.I., T.S. and T.N.; visualization, Y.M.; supervision, H.I., T.S. and T.N.; project administration, T.N.; funding acquisition, K.O. All authors have read and agreed to the published version of the manuscript.

Funding: This research received no external funding.

Institutional Review Board Statement: Not applicable.

Informed Consent Statement: Not applicable.

Conflicts of Interest: The authors declare no conflict of interest.

\section{References}

1. Popovic, M.; Campos-Möller, X.; Schlenker, M.B.; Ahmed, I.I. Efficacy and Safety of Femtosecond Laser-Assisted Cataract Surgery Compared with Manual Cataract Surgery: A Meta-Analysis of 14,567 Eyes. Ophthalmology 2016, 123, 2113-2126. [CrossRef] [PubMed]

2. Ide, T.; Yoo, S.H.; Lee, R.K.; O’Brien, T.P. Allopatric femtosecond laser gas-bubble formation in a closed system. J. Cataract Refract. Surg. 2009, 35, 1619-1622. [CrossRef] [PubMed]

3. Roberts, T.V.; Sutton, G.; Lawless, M.A.; Jindal-Bali, S.; Hodge, C. Capsular block syndrome associated with femtosecond laser-assisted cataract surgery. J. Cataract Refract. Surg. 2011, 37, 2068-2070. [CrossRef] [PubMed]

4. Yoe, R. Hydrorupture of posterior capsule in femtosecond-laser cataract surgery. J. Cataract Refract. Surg. 2012, $38,730$.

5. Sperl, P.; Strohmaier, C.; Kraker, H.; Motloch, K.; Lenzhofer, M.; Moussa, S.; Reitsamer, H.A. Intraocular Pressure Course During the Femtosecond Laser-Assisted Cataract Surgery in Porcine Cadaver Eyes. Investig. Ophthalmol. Vis. Sci. 2017, 58, 6457-6461. [CrossRef] [PubMed]

6. Masuda, Y.; Iwaki, H.; Kato, N.; Watanabe, A.; Takada, A.; Okamoto, T.; Oki, K.; Nakano, T.; Tsuneoka, H. The safety and efficacy of phaco-sleeve irrigation-assisted hydrodissection during femtosecond laser-assisted cataract surgery. Clin. Ophthalmol. 2018, 12, 1829-1835. [CrossRef] [PubMed]

7. Masuda, Y.; Iwaki, H.; Kato, N.; Takahashi, G.; Oki, K.; Tsuneoka, H. Irrigation dynamic pressure-assisted hydrodissection during cataract surgery. Clin. Ophthalmol. 2017, 11, 323-328. [CrossRef] [PubMed]

8. Rossi, M.; Di Censo, F.; Di Censo, M.; Oum, M.A. Changes in Aqueous Humor pH After Femtosecond Laser-Assisted Cataract Surgery. J. Refract. Surg. 2015, 31, 462-465. [CrossRef] [PubMed]

9. Masuda, Y.; Igarashi, T.; Oki, K.; Kobayashi, M.; Takahashi, H.; Nakano, T. Free radical production by femtosecond laser lens irradiation in porcine eyes. J. Cataract Refract. Surg. 2019, 45, 1168-1171. [CrossRef] [PubMed] 